Article

\title{
PlanetCARMA: A New Framework for Studying the Microphysics of Planetary Atmospheres
}

\author{
Erika Barth
}

Southwest Research Institute, Boulder, CO 80302, USA; ebarth@boulder.swri.edu

Received: 1 September 2020; Accepted: 28 September 2020; Published: 6 October 2020

check for updates

\begin{abstract}
The Community Aerosol and Radiation Model for Atmospheres (CARMA) has been updated to apply to atmospheres of the Solar System outside of Earth. CARMA, as its name suggests, is a coupled aerosol microphysics and radiative transfer model and includes the processes of nucleation, condensation, evaporation, coagulation, and vertical transport. Previous model versions have been applied separately to the atmospheres of Solar System bodies and extrasolar planets. The primary advantage to PlanetCARMA is that the core physics routines each reside in their own self-contained modules and can be turned on/off as desired while a separate planet module supplies all the necessary parameters to apply the model run to a particular planet (or planetary body). So a single codebase is used for all planetary studies. PlanetCARMA has also been updated to Fortran 90 modular format. Examples of outer solar system atmosphere applications are shown.
\end{abstract}

Keywords: modeling; aerosols; microphysics

\section{Introduction}

The Community Aerosol and Radiation Model for Atmospheres (CARMA) has been an important tool in understanding the microphysical interactions of gases and particles in the Earth's atmosphere. Versions of this model have also been adapted on an individual basis to solar system terrestrial [1-4], giant [5-7], and extrasolar planet atmospheres [8,9]. Each of the above studies created a separate CARMA code specific to the planetary atmosphere in question. A new CARMA, now called PlanetCARMA, has been developed which allows a single, common codebase to be used for modeling any planetary atmosphere to which CARMA could previously be applied. This is possible because the underlying physical equations and numerical methods have remained unchanged for each atmosphere modeled. Modifications specific to an atmosphere can then be stored in separate modules and the full model compiled for an individual planetary atmosphere. The advantages of this method include more efficient model upkeep; for example, improvements to a common physics algorithm need only be coded once and then are available for all planet modes of execution. Additionally, PlanetCARMA now provides a single codebase for comparison studies of atmospheres, eliminating any biases introduced from using different models to approach the same study.

This paper serves to introduce the new PlanetCARMA framework to the planetary atmospheres community. Section 2 describes the CARMA model in general and previous versons. Section 3 includes changes for implementing PlanetCARMA. A number of results from applying PlanetCARMA to outer planet atmospheres are illustrated in Section 4, including Titan, Saturn, and Pluto. More detailed studies of Titan and Pluto can be found in $[10,11]$, respectively. PlanetCARMA also includes the two stream radiative transfer code from CARMA. This work concentrates on the microphysics portion of the model. 


\section{CARMA}

PlanetCARMA was started from CARMA versions 2.0-2.3. Another version of CARMA (CARMA3.0) has been developed concurrently by [12,13]. CARMA is a 3-D, Eularian model for simulating microphysics and radiative transfer. The aerosol microphysics portion solves the continuity equation:

$$
\begin{array}{r}
\frac{\partial n}{\partial t}=-\frac{\partial}{\partial z}\left(n v_{\text {fall }}\right)-n_{\text {gas }} K_{\text {diff }} \frac{\partial\left[n(v) / n_{\text {gas }}\right]}{\partial z} \\
+\frac{1}{2} \int_{0}^{v} K_{\text {coag }}\left(v^{\prime}, v-v^{\prime}\right) n\left(v^{\prime}\right) n\left(v-v^{\prime}\right) d v^{\prime} \\
-\int_{0}^{\infty} K_{\text {coag }}\left(v^{\prime}, v\right) n(v) n\left(v^{\prime}\right) d v^{\prime} \\
+P(v)-L(v)
\end{array}
$$

for involatile aerosol particles, and

$$
\begin{array}{r}
\frac{\partial n}{\partial t}=-\frac{\partial}{\partial z}\left(n v_{\text {fall }}\right)-n_{\text {gas }} K_{\text {diff }} \frac{\partial\left[n(v) / n_{\text {gas }}\right]}{\partial z} \\
+\frac{1}{2} \int_{0}^{v} K_{\text {coag }}\left(v^{\prime}, v-v^{\prime}\right) n\left(v^{\prime}\right) n\left(v-v^{\prime}\right) d v^{\prime} \\
-\int_{0}^{\infty} K_{\text {coag }}\left(v^{\prime}, v\right) n(v) n\left(v^{\prime}\right) d v^{\prime} \\
+J(v)-\frac{\partial}{\partial v}(G(v) n(v))
\end{array}
$$

for cloud particles.

Both aerosol and cloud particles are transported vertically through sedimentation (regulated by fall velocity, $v_{\text {fall }}$ ) and eddy diffusion (with coefficient, $K_{d i f f}$ ). All particles are also subject to coagulation (with coagulation kernel $K_{\text {coag }}$ ). The production term, $P(v)$, in Equation (1) describes the source term for the involatile particles; these could be supplied by a flux into the model or a production function simulating photochemical production within the model domain. Aerosol particles are lost, $L(v)$ in Equation (1), through nucleation to produce cloud particles, $J(v)$ in Equation (2). Cloud particles then interact with the volatiles through condensational growth and evaporation (regulated by the growth kernel, $G(v)$ ).

CARMA is a bin model. Aerosol particles are stored as a number of radius bins defined from a minimum value and mass ratio between bins. Aerosols are divided into groups which are then subdivided into elements. A group specifies an aerosol type, such as haze particle, dust particle, methane cloud particle, etc. Involatile particle groups generally consist of only one element which represents the number density. Volatile (cloud) groups include the number density element and additional elements to track the mass of the cores. The physical processes in CARMA move mass between size bins (coagulation, condensation, evaporation), between groups (nucleation, coagulation), and between gridboxes (transport).

The algorithms for the CARMA physical processes are described in more detail in [14,15]. A description of each physical process is included below, as well as changes that have been added in the course of planetary modeling and implementation into the PlanetCARMA framework.

\section{New Framework for Planetary Application}

PlanetCARMA is possible because the same basic physical processes apply across all the atmospheres. Physical processes are included in the Common code, which is then linked to a World Module to supply the planetary specifics as further described below. While CARMA was originally a 3-D model and is particularly used as such in the terrestrial community (e.g., [16]), the planetary applications have primarily used it as a 1-D model. As such, the horizontal transport algorithms and 
all $\mathrm{x}$-/y-dimensions have been removed so that PlanetCARMA is a 1-D column model. This change also facilitates driving PlanetCARMA from a 2-D or 3-D dynamic model, such as the Titan or Mars Regional Atmospheric Modeling System (TRAMS, MRAMS), though a description of that work is beyond the scope of this paper. Additionally, while the microphysics part of CARMA was written for cgs units, PlanetCARMA has been converted to mks.

Physical processes in the Common code operate on the atmospheric column and include (1) vertical transport, (2) coagulation, and (3) cloud microphysics.

Vertical transport moves the particles and gases between layers by diffusion (specified by an eddy diffusion equation) and advection (graviational sedimentation, vertical wind). Boundary conditions can be specified to include both advection and diffusion across the external model boundaries or allow a user specified flux. The transport equation is put into Crank-Nicolson form to solve:

$$
a l(k) c(k+1)+b l(k) c(k)+u l(k) c(k-1)=d l(k)
$$

where

$$
\begin{array}{r}
a l(k)=u c *\left(v_{D} \downarrow(k+1)+v_{A} \downarrow(k+1)\right) \\
b l(k)=-\left(u c * \left(v_{D} \downarrow(k)+v_{D} \uparrow(k+1)+\right.\right. \\
\left.\left.v_{A} \downarrow(k)+v_{A} \uparrow(k+1)\right)+d z(k) / d t\right) \\
u l(k)=u c *\left(v_{D} \uparrow(k)+v_{A} \uparrow(k)\right) \\
d l(k)=C(k) *\left(( 1 - u c ) * \left(v_{D} \downarrow(k)+v_{D} \uparrow(k+1)+\right.\right. \\
\left.v_{A} \downarrow(k)+v_{A} \uparrow(k+1)\right) \\
-d z(k) / d t)- \\
(1-u c) *\left(\left(v_{D} \uparrow(k)+v_{A} \uparrow(k)\right) * C_{l}(k)+\right. \\
\left.\left(v_{D} \downarrow(k+1)+v_{A} \downarrow(k+1)\right) * C_{u}(k)\right)-\operatorname{divcor}(k) * d z(k)
\end{array}
$$

The concentrations (particle number or gas mass) are $C$; diffusion and advection fluxes are specified as $v_{D}$ and $v_{A}$, with the arrow indicating direction across the layer boundary; $u c$ is the Courant number; $d z$ is the layer spacing; $d t$ is the timestep; and divcor is a divergence correction term for 1-D modeling. The transport equation and much of the transport algorithms to supply the terms in PlanetCARMA remain unchanged from CARMA.

Coagulation includes brownian and coalescence. The sticking coefficient for the Brownian coagulation kernel can be modified to include the effects of charge on the aerosol particles. This generally serves to decrease the sticking coefficient from unity $\left(c_{\text {stick }}\right.$ in the coagulation kernel, $K_{\text {coag, equation: }}$

$$
\begin{array}{r}
K_{\text {coag }}\left(r_{i}, r_{j}\right)=4 \pi\left(D_{i}+D_{j}\right)\left(r_{i}+r_{j}\right) \zeta \\
\beta \approx \frac{V_{i j}\left(r_{i}+r_{j}\right) c_{\text {stick }}}{4\left(D_{i}+D_{j}\right)}
\end{array}
$$

where, for particles $i$ and $j, r$ is the radius, $D$ is the diffusion coefficient, and $V$ is the thermal velocity. Ref. [17] first adapted the work of [18] to calculate new coagulation kernels for Titan haze particles. Free electrons in Titan's atmosphere attach to the high altitude haze particles. The charge to radius ratio $(p / r)$ is calculated by solving the equation for $x$, which sums over the particle size distribution $(N)$ :

$$
\begin{array}{r}
x \exp -x=\frac{-I}{\pi v_{t h} \frac{N r k_{B} T}{e^{2}} N r^{2}} \\
x=\frac{p_{i}}{r_{i}} \frac{e^{2}}{k_{B} T}
\end{array}
$$


The ionization rate $I$ and the thermal velocity $v_{t h}$ can be calculted from the tables in [18], $e$ is the unit of charge, $k_{B}$ is Boltzmann's constant, and $T$ is the temperature.

$$
c_{\text {stick }}=\exp -\frac{r_{i} * r_{j}(p / r)^{2} e^{2}}{k_{B} T\left(r_{i}+r_{j}\right)}
$$

The resulting sticking coefficient serves to shift the peak of the size distribution for coagulating spherical particles to $\sim 0.1 \mu \mathrm{m}$. Charge is also expected to affect the sticking of aerosol particles in Pluto's atmosphere, but equivalent tables of ionization rates are not known.

The coalescence kernel is calculated from a combination of collision and coalescence efficiencies $\left(E_{\text {coll }}, E_{\text {coal }}\right)$ derived from a table of experiments with water droplets.

$$
K_{\text {coag }}=E_{\text {coll }} E_{c o a l} \pi(r i+r j)^{2}\left(v_{f i}-v_{f j}\right)
$$

where $v_{f}$ are fall velocities, with $r_{i}>r_{j}$. Additional options for calculating the coalescence kernel have been added based on experiments in the fuel industry which employ the Webber number

$$
W e=\frac{\rho_{d r o p}\left(v_{f i}-v_{f j}\right)^{2} 2 r_{i}}{\sigma_{l v}}
$$

$E_{\text {coal }}$ is proportional to the inverse of We. The Weber number compares the inertial force to the surface force, where $\rho_{d r o p}$ is the density of the droplet and $\sigma_{l v}$ is the surface tension. Additional description can be found in [19]. This formulation may be more applicable to the trace hydrocarbon species found in outer planet atmospheres.

The cloud microphysics includes the processes of nucleation, condensation, and evaporation. CARMA includes the nucleation options of droplet activation, droplet freezing, and ice crystal melting, all with a prescribed constant nucleation loss rate. For particles of mixed composition, the volatile core elements can also freeze or melt. PlanetCARMA additionally includes calculating the heterogeneous nucleation rate from vapor deposition [1] and surface diffusion:

$$
J_{\text {het }}=4 \pi^{2} r_{N}^{2} a_{g}^{2} Z \beta c_{1} \exp \left(-\frac{\Delta F}{k T}\right)
$$

Homogeneous nucleation is also included:

$$
J_{\text {hom }}=4 \pi a_{g}^{2} Z \beta n_{1} \exp \left(-\frac{\Delta F}{k T}\right)
$$

$\Delta F$ is the energy barrier to nucleation; $k$ is the Boltzmann constant; $T$ is the temperature; $a_{g}$ is the radius of the germ; $Z$ is the Zeldovich factor; and $r$ is the radius. Using Equation (10) requires calculating the contact parameter, which serves to decrease the energy term from that in Equation (11). The contact parameters is calculated by solving Equation (10) for the case of $J=1$ at the critical saturation, $S_{\text {crit }}$. Few measurements of $S_{\text {crit }}$ exist so PlanetCARMA includes a test routine for calculating a contact parameter from any $S_{\text {crit }}$ value; this is described more fully in [10] and was used to constrain estimates for the nucleation rates for many of the hydrocarbons found in Titan's atmosphere.

An additional source term for particles (added in the same manner as nucleation production) is included from parameterized photochemical production. This follows a gaussian profile and can be applied for both particles and gases. The production rate, $q$, for each altitude layer, $z$, is defined by the altitude of maximum production, $z_{0}$, and the scale height of production, $z_{\mathcal{s}}$. The scale height can differ above and below $z_{0}$.

$$
q=\exp -0.5\left(\left(z-z_{0}\right) / z_{s}\right)^{2}
$$


The World Module supplies the specifics (i.e., numbers, equations, datasets) for using PlanetCARMA to simulate each planetary body. Examples include:

- Define atmosphere input files (pressure-temperature);

- Access molecular weight, viscosity, thermal conductivity for atmospheric composition;

- Eddy diffusion profile, vertical wind speeds, gravity;

- Particle composition and relationships (which volatile species are present, types of particles that serve as cloud condensation nuclei, nucleation rates or contact parameters).

Additionally, the world modules contain subroutines to augment the common physical processes. For example, calculating the decrease in the sticking coefficient for Brownian coagulation of charged haze particles in Titan's atmosphere, parameterizing the photochemical production of haze particles on Titan or Pluto or gases on Saturn, Jupiter, and Venus, and homogeneous nucleation of water ice particles in Saturn's atmosphere.

Other changes between PlanetCARMA and previous CARMA versions include the addition of a namelist, volatiles databases, and radiative transfer databases. The namelist contains five parts, one for model configuration (timestep, domain size, etc.) and two each for microphysics and radiative transfer (general and planet-specific). Toggle switches can be set to "turn on" specific particles or gases to be modeled in a particular simulation as well as the physical processes. In this way, many simulations can be conducted without recompiling the model. The volatiles databases include all information for vapor pressure, latent heat, diffusivity, surface tension, molecular weight, and density for the atmospheric species participating in cloud microphysics. Similar radiative transfer databases supply refractive index values and absorption coefficients.

Below are some examples from running PlanetCARMA for particular planetary atmospheres. The additions to the previous CARMA physics are also highlighted as applicable to each atmosphere.

\section{PlanetCARMA}

\subsection{Titan}

TitanCARMA was originally developed to match a steady-state haze profile to Voyager data and explore the microphysical processes that influenced the haze distribution [17]. The model was initialized with no particles present and haze particles (representing the organic carbon-hydrogen-nitrogen particles seen in the main haze layer) were created each timestep from a gaussian production function that added mass at the rate of methane photochemical destruction. These $0.001 \mu \mathrm{m}$ haze particles then grow up to a few microns in radius due to Brownian coagulation. Coagulation of the haze particles was affected by charge on the particles as described in Section 3. Methane and ethane gases were added to explore the formation of cloud particles composed of multiple volatiles $[1,2]$. These simulations used spherical particles. Subsequent improvements added fractal particle shape and expanded to include over a dozen of the photochemically-produced trace gas species [10].

In the ice condensation studies (Figures 1-4), gases are supplied as a flux at the model top boundary (to simulate transport into the model domain from phtochemical production at higher altitudes). Haze particles from the steady-state model serve as condensation nuclei, with a contact parameter calculated based on $\mathrm{CH}_{4}, \mathrm{C}_{2} \mathrm{H}_{6}$, and $\mathrm{C}_{4} \mathrm{H}_{10}$ lab measurements [20,21]. 


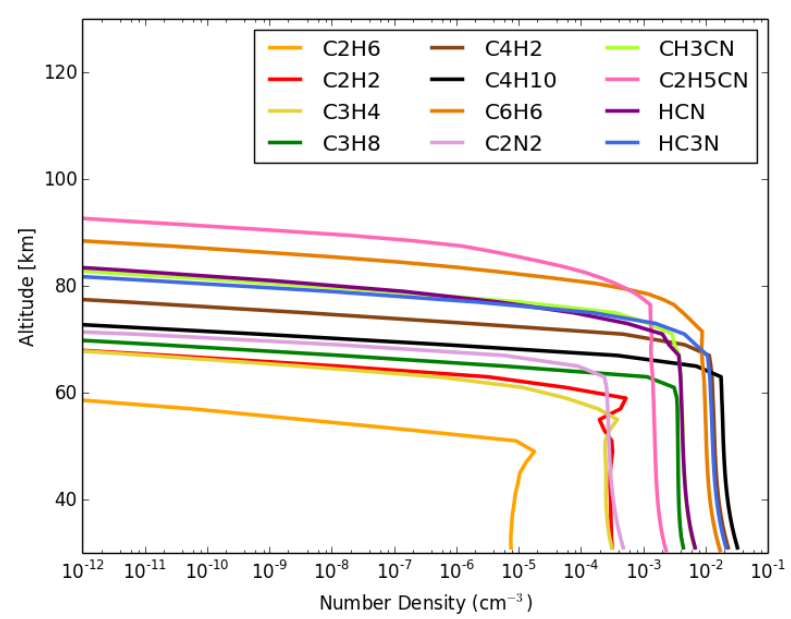

Figure 1. Ice condensation layers formed by many of the hydrocarbon and nitrile trace species found in Titan's atmosphere.

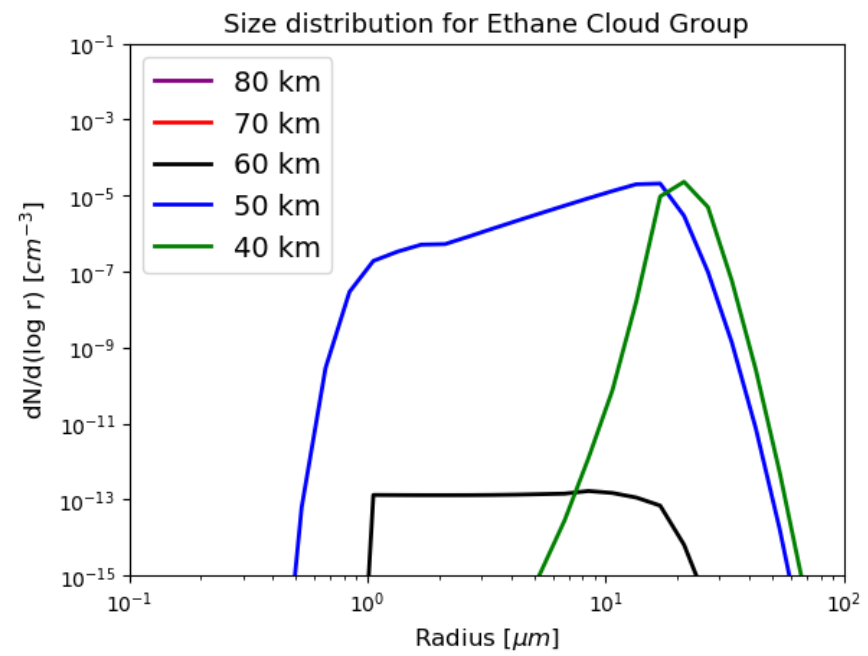

Figure 2. Size distributions at various altitudes of the ethane particles. The low volatility of the ethane vapor pressure at Titan's stratospheric temperatures allows for efficient condensation growth of the ice particles (similar to methane).

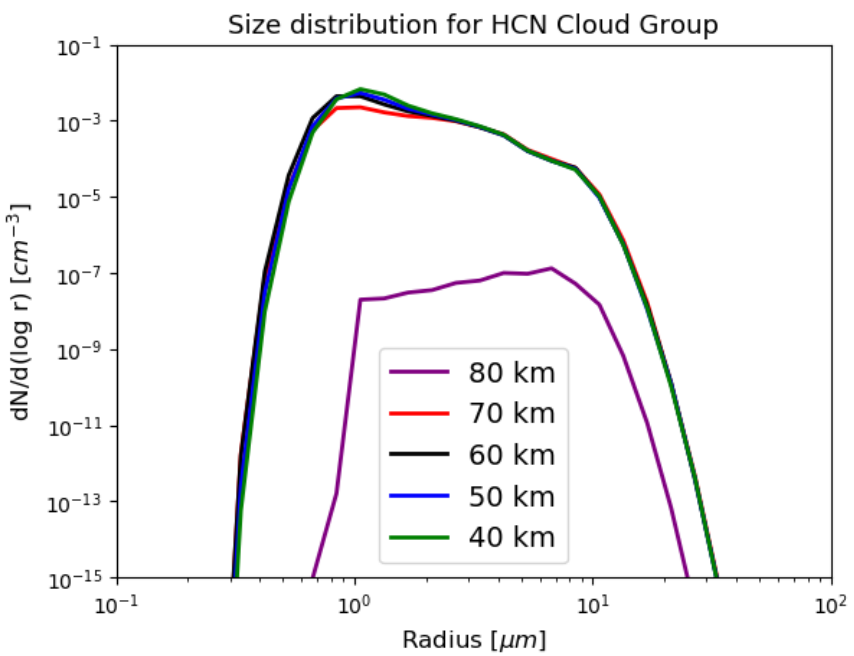

Figure 3. Size distribution of HCN particles in Titan's atmosphere. 


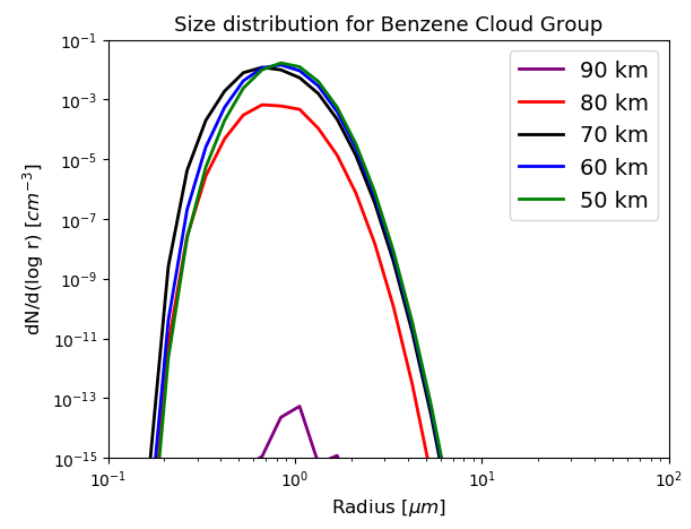

Figure 4. Size distribution of benzene ice particles in Titan's atmosphere.

The layered structure of the cloud particle number density profiles implies that Titan's stratospheric particles are composed of a mixture of ices. Ref. [2] developed the code to model mixtures of methane and ethane ices surrounding a haze CCN. This machinery was ported over to PlanetCARMA, but has not been used in subsequent Titan studies. A future planned development for PlanetCARMA is to rewrite this code to more effciently handle more than two volatiles on a cloud particle.

\subsection{Saturn}

Refs. [6,22] applied previous versions of CARMA to the analysis of Voyager limb images of Uranus and Neptune. Addtionally, ref. [5] modeled the microphysics of aerosol particles in Jupiter's stratosphere and upper troposphere. PlanetCARMA is the first known study applying CARMA microphysics to Saturn. A JupiterCARMA module has been developed as well. SaturnCARMA was configured to explore the makeup of haze particles in the stratosphere. Similar condensation studies to those conducted for Titan are included (Figure 5). However, $\mathrm{H}_{2} \mathrm{O}$ is supplied from impacts and can nucleate homogeneously. These water ice particles could then serve as the condensation nuclei for hydrocarbons deeper in Saturn's stratosphere. Furthermore, on Saturn, photochemical production and loss of gases occurs within the same region of the model domain used for modeling condensation - this differs from Titan where the photochemistry is occuring much higher in the atmosphere and thus gas production can be simulated as a flux into the top boundary of the Titan model. Gaussian production functions parameterize this photochemistry in the Saturn microphysics model, as described above. Figure 6 demonstrates the effects on diacetylene gas $\left(\mathrm{C}_{4} \mathrm{H}_{2}\right)$ when photochemical production and loss through condensation are modeled simultaneously.

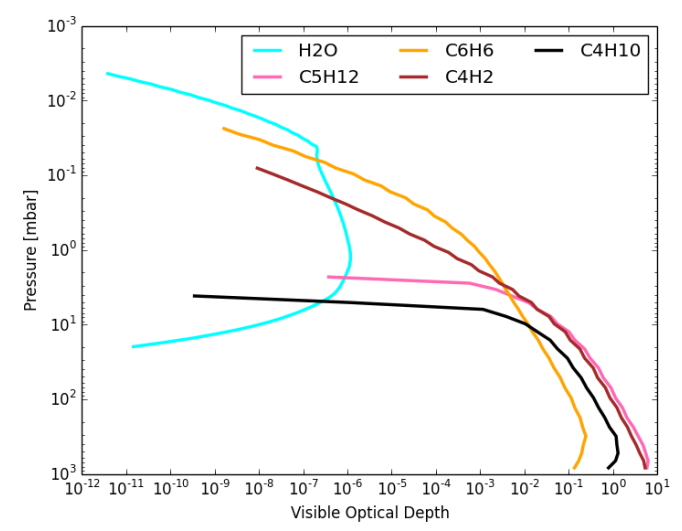

Figure 5. Geometric limit optical depth (per layer) of the ice particles formed in Saturn's atmosphere. Water ice particles can form from either homogeneous nucleation or heterogeneous nucleation onto involatile haze particles. Both types of particles then serve as condensation nuclei for the trace hydrocarbons shown. 


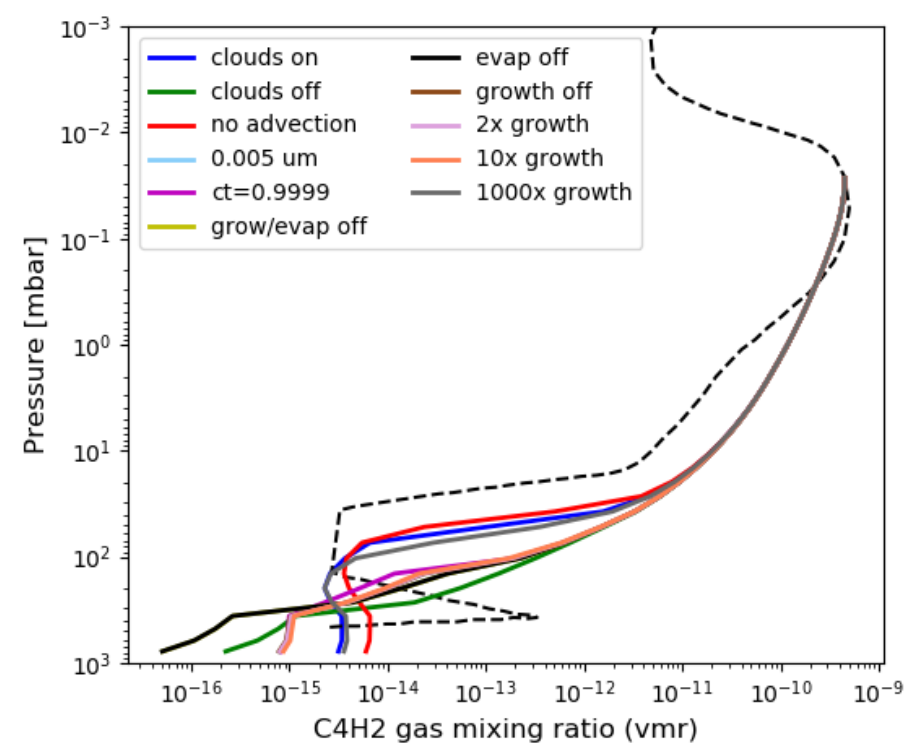

Figure 6. Sensitivity tests showing the effects of different microphysical processes on the $\mathrm{C}_{4} \mathrm{H}_{2}$ gas in Saturn's atmosphere after the 2010 storm. The dashed curve shows the $\mathrm{C}_{4} \mathrm{H}_{2}$ volume mixing ratio modeled by [23] for the aftermath of the storm in May 2011. $\mathrm{C}_{4} \mathrm{H}_{2}$ gas is created each timestep using a gaussian production function to simulate photochemistry. Loss occurs through cloud formation. The spike in $\mathrm{C}_{4} \mathrm{H}_{2}$ below 100 mbar is believed to be due to evaporation of $\mathrm{C}_{4} \mathrm{H}_{2}$ ice particles. This is consistent with microphysics modeling which shows the absence of any spike when the evaporation process is turned off (green-no cloud microphysics, black-no evaporation, yellow-no growth or evaporation). Other tests show how altering cloud parameters affects the amount of evaporated $\mathrm{C}_{4} \mathrm{H}_{2}$ such as changing the minimum radius of cloud particles from $0.1 \mu \mathrm{m}$ to $0.005 \mu \mathrm{m}$, increasing the contact parameter for heterogeneous nucleation from 0.9991 to 0.9999 to make nucleation more efficient, and increasing the condensation rate. The modular structure of PlanetCARMA allows these parameters to be changed with minimal to no changes within the code.

\subsection{Pluto}

PlutoCARMA was developed to explore the condensation of Pluto's atmospheric species. Ref. [24] originally used CARMA to see if aerosols produced from methane photolysis could be responsible for the haze layer indicated in the [25] occultation data. Particles were allowed to grow through coagulation or condensation, though the condensation was parameterized and the cloud microphysics not explicitly treated. More recently, ref. [26] has adapted CARMA 3.0 to model particles making up the high altitude haze layers imaged in the New Horizons pass. Using PlutoCARMA, ref. [11] found that Pluto's haze particles observed by the New Horizons spacecraft are likely to be coated by ices near Pluto's surface. These ices include $\mathrm{CH}_{4}, \mathrm{C}_{2} \mathrm{H}_{6}, \mathrm{C}_{2} \mathrm{H}_{2}, \mathrm{C}_{2} \mathrm{H}_{4}$, and $\mathrm{HCN}$.

Pluto simulations showed that methane along with photochemically produced species detected by the spacecraft are capable of nucleating onto haze particles (presumed similar to Titan's organic haze particles). Similar to the Titan studies, both nucleation and growth modes can be seen in the ethane ice particles. However, condensation is inefficient for HCN near Pluto's surface, Figure 7; particle size is determined by the condensation nuclei.

The Pluto model shares many of the volatile databases as the Titan model since their atmospheres have been found to contain similar species created by the photochemical destruction of nitrogen and methane. Additionally, a CO database was added for Pluto, however given abundance measurements and temperature profiles determined by the New Horizons flyby, it is not likely that $\mathrm{CO}$ ice will condense on its own in the atmosphere (CO is also present in Titan's atmosphere, but is not abundant enough to condense). The main difference between the Pluto and Titan models is that for Pluto 
atmospheric density can be time-dependent due to condensation of $\mathrm{N}_{2}$ onto the surface (similar to $\mathrm{CO}_{2}$ on Mars).

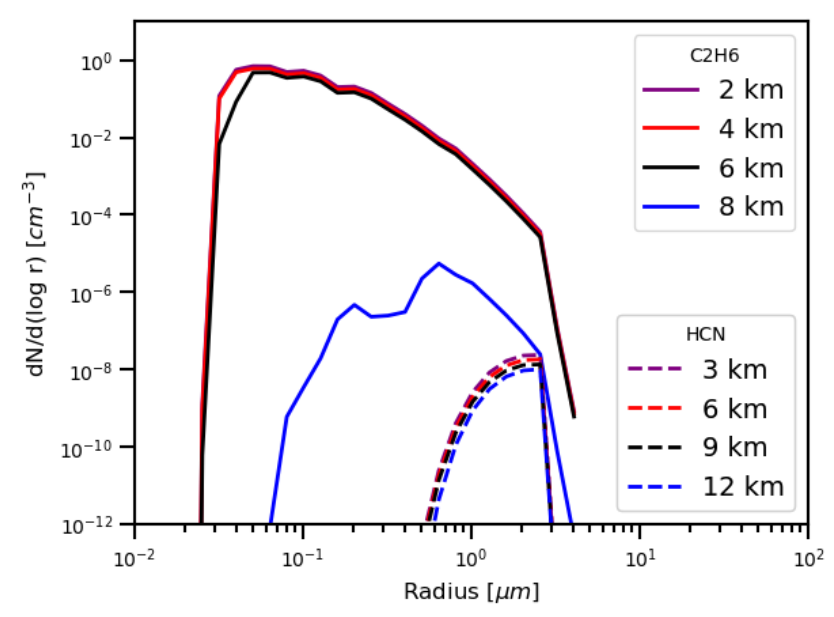

Figure 7. Size distribution of the ethane ice particles formed at the New Horizons entry site. At the altitudes where clouds begin to form, temperatures are warm enough to see both a nucleation and growth mode to the particle sizes. Closer to the surface, further condensation becomes less efficient. Dashed lines show the size distribution of the $\mathrm{HCN}$ ice particles. While nucleation onto pre-existing haze particles can occur, the cold temperatures result in condensation timescales longer than the age of the solar sytem. The resulting HCN ice particle sizes are therefore determined by the size of the haze nuclei and do not show further growth through condensation as they do in Titan's atmosphere. Additionally, HCN only nucleates onto the largest haze particles.

\section{Summary}

A version of the Community Aerosol and Radiation Model for Atmospheres (CARMA) has been updated to make it easily portable to other planetary atmospheres. PlanetCARMA has been applied to studies of the atmospheres of Venus, Mars, Jupiter, Saturn, Titan, and Pluto. Additionally, a database of volatile equations relevant to exoplanet condensing species is currently being added to the code. Sample output for outer solar system studies is provided here-more detailed studies of these atmospheres have been presented elsewhere (e.g., [10,11]). This work serves primarily to present the changes between CARMA and PlanetCARMA as well as present the new algorithms not previously described in other references.

Funding: This research was funded through a combination of NASA research grants (NNX12AM79G, NNX13AK93G, NNX13AL22G) and internal research funding through Southwest Research Institute.

Conflicts of Interest: The author declares no conflict of interest. The funders had no role in the design of the study; in the collection, analyses, or interpretation of data; in the writing of the manuscript, or in the decision to publish the results.

\section{Abbreviation}

The following abbreviation is used in this manuscript:

CARMA Community Aerosol and Radiation Model for Atmospheres

\section{References}

1. Barth, E.L.; Toon, O.B. Microphysical modeling of ethane ice clouds in Titan's atmosphere. Icarus 2003, 162, 94-113. [CrossRef]

2. Barth, E.L.; Toon, O.B. Methane, ethane, and mixed clouds in Titan's atmosphere: Properties derived from microphysical modeling. Icarus 2006, 182, 230-250. [CrossRef] 
3. McGouldrick, K.; Toon, O.B. An investigation of possible causes of the holes in the condensational Venus cloud using a microphysical cloud model with a radiative-dynamical feedback. Icarus 2007, 191, 1-24. [CrossRef]

4. Colaprete, A.; Toon, O.B. Carbon dioxide clouds in an early dense Martian atmosphere. J. Geophys. Res. (Planets) 2003, 108, 5025. [CrossRef]

5. Moreno, F. The structure of the stratospheric aerosol layer in the equatorial and south polar regions of Jupiter. Icarus 1996, 124, 632-644.

6. Pollack, J.B.; Rages, K.; Pope, S.; Tomasko, M.; Romani, P.; Atreya, S. Nature of stratospheric haze on Uranus: Evidence for condensed hydrocarbons. J. Geophys. Res. 1987, 92, 15037-15065.

7. Moses, J.; Fouchet, T.; Bezard, B.; Glandstone, G.; Lellouch, E. Photochemistry and diffusion in Jupiter's stratosphere: Constraints from ISO observations and comparisons with other giant planets. J. Geophys. Res. 2005, 110, E08001. [CrossRef]

8. Marley, M.S.; Ackerman, A.S.; Cuzzi, J.N.; Kitzmann, D. Clouds and Hazes in Exoplanet Atmospheres. In Comparative Climatology of Terrestrial Planets; Mackwell, S.J., Simon-Miller, A.A., Harder, J.W., Bullock, M.A., Eds.; Univ. Arizona Press: Tucson, AZ, USA, 2013; pp. 367-391.

9. Gao, P.; Marley, M.S.; Zahnle, K.; Robinson, T.D.; Lewis, N.K. Sulfur Hazes in Giant Exoplanet Atmospheres: Impacts on Reflected Light Spectra. Astron. J. 2017, 153, 139. [CrossRef]

10. Barth, E.L. Modeling survey of ices in Titan's stratosphere. Planet. Space Sci. 2017, 137, $20-31$.

11. Stern, S.; Kammer, J.; Barth, E.L.; Singer, K.; Lauer, T.; The New Horizons LORRI Instrument Team; The New Horizons Ralph Instrument Team; The New Horizons Atmospheres Investigation Team. Evidence for possible clouds in Pluto's present day atmosphere. AJ 2017, 154, 43.

12. Bardeen, C.; Toon, O.; Jensen, E.; Marsh, D.; Harvey, V. Numerical simulations of the three-dimensional distribution of meteoric dust in the mesosphere and upper stratosphere. J. Geophys. Res. 2008, 113, D17202.

13. Bardeen, C.; Toon, O.B.; Jensen, E.; Hervig, M.E.; Randall, C.E.; Benze, S.; Marsh, D.R.; Merkel, A. Numerical simulations of the three-dimensional distribution of polar mesospheric clouds and comparisons with Cloud Imaging and Particle Size (CIPS) experiment and the Solar Occultation For Ice Experiment (SOFIE) observations. J. Geophys. Res. 2010, 115, D10204. [CrossRef]

14. Turco, R.; Hamill, P.; Toon, O.; Whitten, R.; Kiang, C. A one-dimensional model describing aerosol formation and evolution in the stratosphere: I. Physical processes and mathematical analogs. J. Atmos. Sci. 1979, $36,699-717$.

15. Toon, O.B.; Turco, R.P.; Westphal, D.; Malone, R.; Liu, M.S. A multidimensional model for aerosols: Description of computational analogs. J. Atmos. Sci. 1988, 45, 2123-2143.

16. Colarco, P.; Toon, O.; Reid, J.; Livinston, J.; Russell, P.; Redemann, J.; Schmid, B. Saharan dust transport to the Caribbean during PRIDE: 2. Transport, vertical profiles, and deposition in simulations of in situ and remote sensing observatons. J. Geophys. Res. 2003, 108, 8590.

17. Toon, O.B.; McKay, C.P.; Griffith, C.A.; Turco, R.P. A physical model of Titan's aerosols. Icarus 1992, 95, $24-53$. [CrossRef]

18. Borucki, W.J.; Levin, Z.; Whitten, R.C.; Keesee, R.G.; Capone, L.A.; Summers, A.L.; Toon, O.B.; Dubach, J. Predictions of the electrical conductivity and charging of the aerosols in Titan's atmosphere. Icarus 1987, 72, 604-622.

19. Barth, E.L.; Rafkin, S.C. Convective cloud heights as a diagnostic for methane environment on Titan. Icarus 2010, 206, 467-484. [CrossRef]

20. Curtis, D.B.; Glandorf, D.L.; Toon, O.B.; Tolbert, M.A.; McKay, C.P.; Khare, B.N. Laboratory studies of butane nucleation on organic haze particles: Application to Titan's clouds. J. Phys. Chem. A 2005, 109, 1382-1390. [CrossRef]

21. Curtis, D.B.; Hatch, C.D.; Hasenkopf, C.A.; Toon, O.B.; Tolbert, M.A.; McKay, C.P.; Khare, B.N. Laboratory studies of methane and ethane adsorption onto organic particles: Application to Titan's clouds. Icarus 2008, 195, 792-801. [CrossRef]

22. Moses, J.; Rages, K.; Pollack, J. An analysis of Neptune's stratospheric haze using high-phase-angle Voyager images. Icarus 1995, 113, 232-266. [CrossRef]

23. Moses, J.I.; Armstrong, E.S.; Fletcher, L.N.; Friedson, A.J.; Irwin, P.G.J.; Sinclair, J.A.; Hesman, B.E. Evolution of stratospheric chemistry in the Saturn storm beacon region. Icarus 2015, 261, 149-168. [CrossRef] [PubMed] 
24. Stansberry, J.A.; Lunine, J.I.; Tomasko, M.G. Upper limits on possible photochemical hazes on Pluto. Geophys. Res. Lett. 1989, 16, 1221-1224. [CrossRef]

25. Elliot, J.L.; Dunham, E.W.; Bosh, A.S.; Slivan, S.M.; Young, L.A.; Wasserman, L.H.; Millis, R.L. Pluto's Atmosphere. Icarus 1989, 77, 148-170. [CrossRef]

26. Gao, P.; Fan, S.; Wong, M.L.; Liang, M.C.; Shia, R.L.; Kammer, J.A.; Yung, Y.L.; Summers, M.E.; Gladstone, G.R.; Young, L.A.; et al. Constraints on the microphysics of Pluto's photochemical haze from New Horizons observations. Icarus 2017, 287, 116-123. [CrossRef] 